\title{
An Efficient and Accurate Spectral Method for Acoustic Scattering in Elliptic Domains
}

\author{
Qirong Fang ${ }^{1}$, Jie Shen ${ }^{1, *}$ and Li-Lian Wang ${ }^{2}$ \\ ${ }^{1}$ Department of Mathematics, Purdue University, West Lafayette, IN 47907, USA. \\ ${ }^{2}$ Division of Mathematical Sciences, School of Physical and Mathematical \\ Sciences, Nanyang Technological University, 637616, Singapore.
}

Received 21 August 2008; Accepted (in revised version) 23 January 2009

\begin{abstract}
An efficient and accurate method for solving the two-dimensional Helmholtz equation in domains exterior to elongated obstacles is developed in this paper. The method is based on the so called transformed field expansion (TFE) coupled with a spectral-Galerkin solver for elliptical domain using Mathieu functions. Numerical results are presented to show the accuracy and stability of the proposed method.
\end{abstract}

AMS subject classifications: 78A45, 65N35, 35J05

Key words: Acoustic scattering, high-order methods, boundary perturbations, spectral-Galerkin methods.

\section{Introduction}

Many scientific and engineering applications require fast and accurate numerical approximation of acoustic and electromagnetic scattering that returns from irregular obstacles. Although the governing equation is linear, its numerical approximation presents a number of notorious difficulties: (i) the problem is set in an unbounded exterior domain, making it difficult to obtain accurate approximations when an artificial boundary is introduced; (ii) the problem is indefinite, making it difficult to design efficient iterative methods; and (iii) the solution is highly oscillatory when the incoming wave has high frequencies, making it inefficient to use low-order finite difference or finite element methods. A wide variety of numerical methods have been proposed to deal with these difficulties (cf. the review papers $[14,19]$ and the references therein). A particularly compelling class of methods are based on the boundary perturbation technique originated from the work of Rayleigh [13] and Rice [15], and we refer to [2-4] for some recent developments in this direction. More recently, a robust and accurate numerical method based on the transformed field expansion and a fast spectral-Galerkin solver is proposed for two- and

*Corresponding author. Email addresses: qfang@math.purdue.edu (Q. Fang), shen@math.purdue.edu (J. Shen), lilian@ntu.edu.sq (L. Wang) 
three-dimensional acoustic scattering $[5,9,11,12]$. The method has proven to be very efficient for obstacles that can be considered as a perturbation of a disk in 2-D or a sphere in 3-D. While in principle the algorithms in [5,9] can be applied to elongated scatters (e.g., submarines and airfoils), which are found in many important applications, it may not be computationally efficient to do so due to the fact that large artificial boundaries are needed to enclosed the elongated obstacles. In such cases, it is more appropriate to use elliptic and ellipsoidal artificial surfaces to truncate the unbounded computational domains.

The purpose of this paper is to develop an efficient and accurate numerical method for the acoustic scattering from an elongated obstacle. The basic idea is to consider an elongated obstacle as a perturbation of ellipse in 2-D and of ellipsoid in 3-D, use a larger ellipse or ellipsoid to enclose the obstacle and reduce the problem to a bounded domain through the Dirichlet-to-Neumann mapping, and then develop an efficient and accurate spectral method for the reduced equation in the separated elliptic domain.

While spectral methods for partial differential equations in circular and spherical domains have been well developed, their applications to elliptical domains have received very little attention. The main reason is that the separation of variables in elliptical domains leads to Mathieu functions in 2-D and spheroidal wave functions in 3-D. However, the use of elliptic coordinates and Mathieu functions introduces significant difficulties in both analysis and implementation. Although the Mathieu functions have been the subjects of many studies (cf. $[1,7,8]$ ), most of which are concerned with their classical properties such as identities, recurrence and asymptotic relations. As far as we know, there were essentially no results on their approximation properties in Sobolev spaces which are required for numerical analysis of spectral methods using these special functions. In a very recent paper [18], two of the authors made a systematical study for the approximation properties of Mathieu functions and applied them to study the elliptic equations in a bounded separable elliptic domain. The analytical and numerical results presented in [18] indicate that Mathieu functions have nice approximation properties similar to those enjoyed by classical trigonometric polynomials and are suitable for numerical approximation of PDEs in elliptic domains. Hence, we shall use Mathieu functions as basis functions for the spectral-Galerkin solver in our scheme.

The rest of the paper is organized as follow. In Section 2, we describe the governing equation for acoustic scattering in exterior domains with elliptical coordinates and use the Dirichlet-to-Neumann mapping to reduce the problem to a bounded domain. We derive in Section 3 the transformed field expansion in the elliptical coordinates. Then, we construct a spectral-Galerkin method for solving the reduced Helmtoltz problem in a regular elliptical domain. We present some illustrative numerical results in Section 5 and conclude with some remarks in the last section.

\section{Governing equation and Dirichlet-to-Neumann mapping}

\subsection{Governing equation}

Consider a two-dimensional time-harmonic acoustic plane wave 


$$
\tilde{u}_{i}(r, \theta, t)=e^{i \omega t} u_{i}(r, \theta)=e^{i \omega t} e^{i r(\alpha \cos (\theta)-\beta \sin (\theta))}
$$

incident upon a bounded obstacle $\Sigma$. It generates a scattered field

$$
\tilde{u}_{s}(r, \theta, t)=e^{i \omega t} u_{s}(r, \theta)
$$

satisfying the Helmholtz equation

$$
\Delta u_{s}+k^{2} u_{s}=0 \quad \text { in } \quad \Omega=\mathbb{R}^{2} \backslash \bar{\Sigma},
$$

along with the Sommerfeld radiation boundary condition at infinity. In the above, $k=$ $\sqrt{\alpha^{2}+\beta^{2}}$ is the wave number.

In [9], an efficient spectral method was proposed to solve this problem, which was based on a transformed field expansion (TFE) approach and a fast spectral-Galerkin solver in the circular domain. The method is suitable when the obstacle is a "small" perturbation of the circle. When the obstacle has an elongated shape (e.g., a submarine), the method in [9] will not be efficient as a rather large artificial computational domain will have to be used. In this case, it is more desirable to truncate the unbounded domain into an ellipse, and develop the spectral solver in elliptic coordinates:

$$
x=c \cosh \mu \cos \theta, \quad y=c \sinh \mu \sin \theta,
$$

where $2 c$ is the focal distance. Note that curves of constant $\mu$ are all ellipses and the curves of constant $\theta$ are hyperbolas with foci $x= \pm c$ along the $x$-axis. To simplify the notation, we take $c=1$ hereafter.

Under the elliptic coordinates, the domain $\Omega$ can be expressed as

$$
\Omega=\{(\mu, \theta): \mu>a+g(\theta), \theta \in[0,2 \pi)\} .
$$

Denoting $v(\mu, \theta):=u_{s}(x, y)$, the Helmholtz equation (2.3) under the elliptic coordinates becomes

$$
\frac{1}{\frac{1}{2} \cosh (2 \mu)-\frac{1}{2} \cos (2 \theta)}\left(\partial_{\mu}^{2} v+\partial_{\theta}^{2} v\right)+k^{2} v=0, \quad(\mu, \theta) \in \Omega,
$$

along with a boundary condition at $\partial \Omega$, the periodic boundary condition in $\theta$, and the Sommerfeld radiation condition at infinity, namely,

$$
\begin{aligned}
& v(a+g(\theta), \theta)=\xi(\theta), \quad v(\mu, \theta)=v(\mu, \theta+2 \pi), \\
& \lim _{\mu \rightarrow \infty} \mu^{1 / 2}\left(\partial_{\mu} v-i k v\right)=0 .
\end{aligned}
$$

Note that, to fix the idea, we prescribed in (2.6) a Dirichlet (sound-soft) boundary condition on the obstacle, although a Neumann (sound-hard) boundary condition can also be used.

A main difficulty for solving the problem (2.5)-(2.7) is that the domain $\Omega$ is unbounded. However, it is well-known that the solution of the Helmholtz equation (2.5) in the far field can be exactly expressed by expansions in Mathieu functions, leading to an exact expression of the so called Dirichlet-to-Neumann (DtN) operator (cf. [6]), which allows us to reduce the system to a bounded domain. 


\subsection{Mathieu functions}

The Mathieu functions arise when one applies the separation of variables approach to (2.5). More precisely, setting $v(\mu, \theta)=R(\mu) \Phi(\theta)$, we find that $\Phi(\theta)$ satisfies the angular Mathieu equation:

$$
\frac{d^{2} \Phi}{d \theta^{2}}+(a-2 q \cos 2 \theta) \Phi=0
$$

and $R(\mu)$ satisfies the radial Mathieu equation:

$$
\frac{d^{2} R}{d \mu^{2}}-(a-2 q \cosh 2 \mu) R=0,
$$

where $a$ is the separation constant, and the parameter $q=c^{2} k^{2} / 4$.

The angular Mathieu equation (2.8) supplemented with a periodic boundary condition admits two families of linearly independent periodic solutions (eigenfunctions), namely the even and the odd Mathieu functions of order $m$ :

$$
\Phi_{m}(\theta ; q)= \begin{cases}c e_{m}(\theta ; q), & m=0,1, \cdots, \\ s e_{m}(\theta ; q), & m=1,2, \cdots .\end{cases}
$$

Similarly, the radial Mathieu equation (2.9) admits two families of linearly independent solutions, namely the even and the odd Mathieu-Hankel functions of order $m$ :

$$
R_{m}(\mu ; q)= \begin{cases}M c_{m}(\mu ; q), & m=0,1, \cdots, \\ M s_{m}(\mu ; q), & m=1,2, \cdots .\end{cases}
$$

Hence, the general solution of (2.5) can be expressed as

$$
v(\mu, \theta)=\sum_{i=0}^{\infty} \alpha_{i} M c_{i}(\mu ; q) c e_{i}(\theta ; q)+\sum_{i=1}^{\infty} \beta_{i} M s_{i}(\mu ; q) s e_{i}(\theta ; q) .
$$

In this context, the Mathieu-Hankel functions $\left\{M c_{i}, M s_{i}\right\}$ are of the third kind.

We observe that the above expansion is reminiscent to the Fourier-Hankel expansion for the solution of the Helmholtz equation in polar coordinates. Here, the angular Mathieu functions play the role of Fourier series. Indeed, the set of Mathieu functions $\left\{c e_{m}, s e_{m+1}\right\}_{m=0}^{\infty}$ forms a complete orthogonal system in $L^{2}(0,2 \pi)$,

$$
\int_{0}^{2 \pi} c e_{m}(\theta ; q) c e_{n}(\theta ; q) d \theta=\int_{0}^{2 \pi} s e_{m}(\theta ; q) s e_{n}(\theta ; q) d \theta= \begin{cases}\pi, & \text { if } m=n \\ 0, & \text { if } m \neq n\end{cases}
$$

and

$$
\int_{0}^{2 \pi} c e_{m}(\theta ; q) s e_{n}(\theta ; q) d \theta=0, \quad \forall m \geq 0, n \geq 1 .
$$

The interested readers may refer to [8] for more properties of the Mathieu functions. 


\subsubsection{DtN mapping}

With the help of (2.12) and (2.13), we can determine the DtN mapping explicitly. We truncate the domain at $\mu=b>a+|g|_{L^{\infty}}$. Then, given $v(b, \theta)$, we can write the expansion

$$
v(b, \theta)=\psi(\theta)=\sum_{i=0}^{\infty} a_{i} c e_{i}(\theta ; q)+\sum_{i=1}^{\infty} b_{i} s e_{i}(\theta ; q)
$$

Taking $\mu=b$ in (2.12) and comparing with (2.15), we can determine the coefficients $\left\{\alpha_{i}, \beta_{i}\right\}$ in (2.12) uniquely. Consequently, we have

$$
v(\mu, \theta)=\sum_{i=0}^{\infty} a_{i} \frac{M c_{i}(\mu ; q)}{M c_{i}(b ; q)} c e_{i}(\theta ; q)+\sum_{i=1}^{\infty} b_{i} \frac{M s_{i}(\mu ; q)}{M s_{i}(b ; q)} s e_{i}(\theta ; q) .
$$

Therefore, we define the DtN operator $T$ by

$$
T v \equiv \partial_{\mu} v(b, \theta)=\sum_{i=0}^{\infty} a_{i} \frac{M c_{i}^{\prime}(b ; q)}{M c_{i}(b ; q)} c e_{i}(\theta ; q)+\sum_{i=1}^{\infty} b_{i} \frac{M s_{i}^{\prime}(b ; q)}{M s_{i}(b ; q)} s e_{i}(\theta ; q),
$$

which maps Dirichlet data, $\psi$, to Neumann data, $\left.\partial_{\mu} v\right|_{\mu=b}$ (cf. [6]). Thus, the system (2.5)-(2.7) can be equivalently restated as

$$
\begin{aligned}
& \partial_{\mu}^{2} v+\partial_{\theta}^{2} v+\frac{1}{2} k^{2}(\cosh (2 \mu)-\cos (2 \theta)) v=0, \quad(\mu, \theta) \in \Omega_{a+g, b} \\
& v(a+g(\theta), \theta)=\xi(\theta), \quad v(\mu, \theta)=v(\mu, \theta+2 \pi) \\
& \partial_{\mu} v(b, \theta)-T v(b, \theta)=0
\end{aligned}
$$

where

$$
\Omega_{a+g, b}=\{(\mu, \theta): a+g(\theta)<\mu<b, 0 \leq \theta<2 \pi\} .
$$

The rest of the paper is devoted to developing and testing an efficient and accurate spectral approximation to this problem.

\section{Transformed field expansions}

\subsection{Transformation to a separable domain}

While Eq. (2.17) is set on a bounded domain, it is still not suitable for spectral methods as the domain $\Omega_{a+g, b}$ is in general not separable. In order to develop an effective spectral method, it is necessary to transform the domain to a separable one. This can be done with the change of variables

$$
\mu^{\prime}=\frac{(b-a) \mu-b g(\theta)}{(b-a)-g(\theta)}=\frac{d \mu-b g}{d-g}, \quad \theta^{\prime}=\theta,
$$

where $d=b-a$, which maps $\Omega_{a+g, b}$ to the elliptic annulus $\Omega_{a, b}$. Next, we need to rewrite (2.17) in these transformed coordinates $\left(\mu^{\prime}, \theta^{\prime}\right)$. 
By using the relations

$$
\partial_{\theta}=\frac{\partial \mu^{\prime}}{\partial \theta} \partial_{\mu^{\prime}}+\partial_{\theta^{\prime}}, \quad \partial_{\mu}=\frac{\partial \mu^{\prime}}{\partial \mu} \partial_{\mu^{\prime}}
$$

it is easy to see that

$$
\begin{aligned}
& (d-g(\theta)) \partial_{\theta}=(d-g(\theta)) \partial_{\theta^{\prime}}-B\left(\mu^{\prime}, \theta^{\prime}\right) \partial_{\mu^{\prime}} \\
& (d-g(\theta)) \partial_{\mu}=d \partial_{\mu^{\prime}} \\
& d \mu=d \mu^{\prime}+A\left(r^{\prime}, \theta^{\prime}\right)
\end{aligned}
$$

where

$$
A\left(r^{\prime}, \theta^{\prime}\right)=g\left(\theta^{\prime}\right)\left(b-r^{\prime}\right), \quad B\left(r^{\prime}, \theta^{\prime}\right)=\partial_{\theta^{\prime}} A .
$$

We first deal with (2.17a). Multiplying $(d-g)^{2}$ to (2.17a), we find

$$
0=(d-g)^{2} \partial_{\mu}^{2} v+(d-g)^{2} \partial_{\theta}^{2} v+\frac{1}{2} k^{2}(d-g)^{2}(\cosh (2 \mu)-\cos (2 \theta)) v .
$$

Denoting $u\left(r^{\prime}, \theta^{\prime}\right)=v\left(r^{\prime}+A / d, \theta^{\prime}\right)$, and using (3.1), we find

$$
\begin{aligned}
(d-g)^{2} \partial_{\mu}^{2} v & =(d-g) \partial_{\mu}\left((d-g) \partial_{\mu} v\right) \\
& =(d-g) \partial_{\mu}\left(d \partial_{\mu^{\prime}} u\right)=d^{2} \partial_{\mu^{\prime}}^{2} u,
\end{aligned}
$$

and

$$
\begin{aligned}
& (d-g)^{2} \partial_{\theta}^{2} v \\
= & (d-g) \partial_{\theta}\left[(d-g) \partial_{\theta} v\right]+\partial_{\theta} g(d-g) \partial_{\theta} v \\
= & {\left[(d-g) \partial_{\theta^{\prime}}-B \partial_{\mu^{\prime}}\right]\left[(d-g) \partial_{\theta^{\prime}}-B \partial_{\mu^{\prime}}\right] u+\partial_{\theta^{\prime}} g\left[(d-g) \partial_{\theta^{\prime}}-B \partial_{\mu^{\prime}}\right] u } \\
= & (d-g)^{2} \partial_{\theta^{\prime}}^{2} u-(d-g)\left(\partial_{\theta^{\prime}} g\right) \partial_{\theta^{\prime}} u-(d-g) \partial_{\theta^{\prime}}\left[B \partial_{\mu^{\prime}} u\right]-B(d-g) \partial_{\mu^{\prime}} \partial_{\theta^{\prime}} u \\
& +B \partial_{\mu^{\prime}}\left[B \partial_{\mu^{\prime}} u\right]+(d-g)\left(\partial_{\theta^{\prime}} g\right) \partial_{\theta^{\prime}} u-\left(\partial_{\theta^{\prime}} g\right) B \partial_{\mu^{\prime}} u .
\end{aligned}
$$

Moreover, we have

$$
\begin{aligned}
\frac{1}{2} k^{2}(d-g)^{2} \cosh (2 \mu) v & =\frac{1}{4} k^{2}(d-g)^{2}\left(e^{2 \mu}+e^{-2 \mu}\right) v \\
& =\frac{1}{4} k^{2}(d-g)^{2}\left(e^{2 \mu^{\prime}+\frac{2 A}{d}}+e^{-2 \mu^{\prime}-\frac{2 A}{d}}\right) u \\
& =\frac{1}{4} k^{2}(d-g)^{2}\left(e^{2 \mu^{\prime}} e^{\frac{2 A}{d}}+e^{-2 \mu^{\prime}} e^{-\frac{2 A}{d}}\right) u
\end{aligned}
$$

Denoting

$$
\begin{aligned}
& A_{1}=\frac{2 A}{d}+\frac{\left(\frac{2 A}{d}\right)^{2}}{2 !}+\frac{\left(\frac{2 A}{d}\right)^{3}}{3 !}+\cdots, \\
& A_{2}=-\frac{2 A}{d}+\frac{\left(\frac{2 A}{d}\right)^{2}}{2 !}-\frac{\left(\frac{2 A}{d}\right)^{3}}{3 !}+\cdots,
\end{aligned}
$$


we have

$$
e^{\frac{2 A}{d}}=1+A_{1}, \quad e^{-\frac{2 A}{d}}=1+A_{2},
$$

and

$$
\begin{aligned}
& \frac{1}{2} k^{2}(d-g)^{2} \cosh (2 \mu) v \\
= & \frac{1}{4} k^{2}(d-g)^{2}\left(e^{2 \mu^{\prime}}+e^{-2 \mu^{\prime}}\right) u+\frac{1}{4} k^{2}(d-g)^{2}\left(e^{2 \mu^{\prime}} A_{1}+e^{-2 \mu^{\prime}} A_{2}\right) u .
\end{aligned}
$$

Collecting the above relations into (3.3), the original equation (2.17a) can be written as

$$
\partial_{\mu^{\prime}}^{2} u+\partial_{\theta^{\prime}}^{2} u+\frac{1}{2} k^{2}\left(\cosh \left(2 \mu^{\prime}\right) u-\cos \left(2 \theta^{\prime}\right)\right) u=F,
$$

where $F$ contains all the extra terms due to the transformation and is given by

$$
\begin{aligned}
-d^{2} F= & -2 d g \partial_{\theta^{\prime}}^{2} u+g^{2} \partial_{\theta^{\prime}}^{2} u-(d-g) \partial_{\theta^{\prime}}\left[B \partial_{\mu^{\prime}} u\right] \\
& -B(d-g) \partial_{\mu^{\prime}} \partial_{\theta^{\prime}} u+B \partial_{\mu^{\prime}}\left[B \partial_{\mu^{\prime}} u\right] \\
& -\left(\partial_{\theta^{\prime}} g\right) B \partial_{\mu^{\prime}} u+k^{2} d g \cos \left(2 \theta^{\prime}\right) u-\frac{1}{2} k^{2} g^{2} \cos \left(2 \theta^{\prime}\right) u-k^{2} d g \cosh \left(2 \mu^{\prime}\right) u \\
& +\frac{1}{2} k^{2} g^{2} \cosh \left(2 \mu^{\prime}\right) u+\frac{1}{4} k^{2}(d-g)^{2}\left(e^{2 \mu^{\prime}} A_{1}+e^{-2 \mu^{\prime}} A_{2}\right) u .
\end{aligned}
$$

Now multiplying (2.17c) by $(d-g)$ and using (3.1), we find

$$
\begin{aligned}
0 & =(d-g) \partial_{\mu} v(b, \theta)-(d-g) T v(b, \theta) \\
& =d \partial_{\mu^{\prime}} u\left(b, \theta^{\prime}\right)-d \operatorname{Tu}\left(b, \theta^{\prime}\right)+g\left(\theta^{\prime}\right) \operatorname{Tu}\left(b, \theta^{\prime}\right) .
\end{aligned}
$$

Therefore, $(2.17 \mathrm{c})$ is transformed into

$$
\partial_{\mu^{\prime}} u\left(b, \theta^{\prime}\right)-T u\left(b, \theta^{\prime}\right)=J\left(\theta^{\prime}\right),
$$

where

$$
J\left(\theta^{\prime}\right)=-\frac{1}{d} g\left(\theta^{\prime}\right) T u\left(b, \theta^{\prime}\right) .
$$

Collecting these transformations, we find that the transformed field $u$, upon dropping primes, satisfies

$$
\begin{aligned}
& \partial_{\mu}^{2} u+\partial_{\theta}^{2} u+\frac{1}{2} k^{2}(\cosh (2 \mu) u-\cos (2 \theta)) u=F, \quad(\mu, \theta) \in \Omega_{a, b}, \\
& u(a, \theta)=\xi(\theta), \quad u(\mu, \theta)=u(\mu, \theta+2 \pi), \\
& \partial_{\mu} u(b, \theta)-T u(b, \theta)=J(\theta)
\end{aligned}
$$

where $F$ and $J(\theta)$ are given by (3.5) and (3.7), respectively. 


\subsection{Boundary perturbation}

While Eq. (3.8) is set on a separable domain, it is still challenging to solve it numerically due to (i) the non-constant coefficients in $F$ which prevents a feasible direct solution, and (ii) its indefiniteness makes it difficult to design an efficient iterative scheme.

Therefore, similar to $[5,9]$, we resort to a perturbation approach. More precisely, we write $g(\theta)=\varepsilon f(\theta)$ and expand the solution $u$ of (3.8) as

$$
u(\mu, \theta ; \varepsilon)=\sum_{n=0}^{\infty} u_{n}(\mu, \theta) \varepsilon^{n}
$$

Inserting the above in (3.8), it is straightforward, albeit tedious, to derive the following recursions for $\left\{u_{n}\right\}$ :

$$
\begin{aligned}
& \partial_{\mu}^{2} u_{n}+\partial_{\theta}^{2} u_{n}+\frac{1}{2} k^{2}(\cosh (2 \mu)-\cos (2 \theta)) u_{n}=F_{n},(\mu, \theta) \in \Omega_{a, b}, \\
& u_{n}(a, \theta)=\delta_{n, 0} \xi(\theta), \quad u_{n}(\mu, \theta)=u_{n}(\mu, \theta+2 \pi), \\
& \partial_{\mu} u_{n}(b, \theta)-T u_{n}(b, \theta)=J_{n}(\theta),
\end{aligned}
$$

where $J_{n}=-(f / d) T u_{n-1}(b, \theta)$, and

$$
\begin{aligned}
-d^{2} F_{n}= & -2 d g \partial_{\theta}^{2} u_{n-1}+g^{2} \partial_{\theta}^{2} u_{n-2}-d \partial_{\theta}\left[B \partial_{\mu} u_{n-1}\right]+g \partial_{\theta}\left[B \partial_{\mu} u_{n-2}\right]-d B \partial_{\mu} \partial_{\theta} u_{n-1} \\
& +g B \partial_{\mu} \partial_{\theta} u_{n-2}+B \partial_{\mu}\left[B \partial_{\mu} u_{n-2}\right]-\left(\partial_{\theta} g\right) B \partial_{\mu} u_{n-2}+k^{2} d g \cos (2 \theta) u_{n-1} \\
& -\frac{1}{2} k^{2} g^{2} \cos (2 \theta) u_{n-2}-k^{2} d g \cosh (2 \mu) u_{n-1}+\frac{1}{2} k^{2} g^{2} \cosh (2 \mu) u_{n-2} \\
& +\frac{1}{4} k^{2} d^{2} e^{2 \mu}\left(\frac{\frac{2 A}{d}}{1 !} u_{n-1}+\frac{\left(\frac{2 A}{d}\right)^{2}}{2 !} u_{n-2}+\cdots+\frac{\left(\frac{2 A}{d}\right)^{n}}{n !} u_{0}\right) \\
& +\frac{1}{4} k^{2} d^{2} e^{-2 \mu}\left(-\frac{\frac{2 A}{d}}{1 !} u_{n-1}+\frac{\left(\frac{2 A}{d}\right)^{2}}{2 !} u_{n-2}+\cdots+(-1)^{n} \frac{\left(\frac{2 A}{d}\right)^{n}}{n !} u_{0}\right) \\
& -\frac{1}{2} k^{2} d g e^{2 \mu}\left(\frac{\frac{2 A}{d}}{1 !} u_{n-2}+\frac{\left(\frac{2 A}{d}\right)^{2}}{2 !} u_{n-3}+\cdots+\frac{\left(\frac{2 A}{d}\right)^{n-1}}{(n-1) !} u_{0}\right) \\
& -\frac{1}{2} k^{2} d g e^{-2 \mu}\left(-\frac{\frac{2 A}{d}}{1 !} u_{n-2}+\frac{\left(\frac{2 A}{d}\right)^{2}}{2 !} u_{n-3}+\cdots+(-1)^{n-1} \frac{\left(\frac{2 A}{d}\right)^{n-1}}{(n-1) !} u_{0}\right) \\
& +\frac{1}{4} k^{2} g^{2} e^{2 \mu}\left(\frac{\frac{2 A}{d}}{1 !} u_{n-3}+\frac{\left(\frac{2 A}{d}\right)^{2}}{2 !} u_{n-4}+\cdots+\frac{\left(\frac{2 A}{d}\right)^{n-2}}{(n-2) !} u_{0}\right) \\
& +\frac{1}{4} k^{2} g^{2} e^{-2 \mu}\left(-\frac{\frac{2 A}{d}}{1 !} u_{n-3}+\frac{\left(\frac{2 A}{d}\right)^{2}}{2 !} u_{n-4}+\cdots+(-1)^{n-2} \frac{\left(\frac{2 A}{d}\right)^{n-2}}{(n-2) !} u_{0}\right) .
\end{aligned}
$$

In the above we adopt the convention that terms with subscripts are set to zero. We observe that $F_{n}$ involves solutions at all previous iterations as opposed to only four previous iterates in the circular or spherical case $[5,9]$. 
It is clear that for smooth $f(\theta)$ there exists $\varepsilon_{0}>0$ such that the Taylor expansion (3.9) converges for all $\varepsilon \leq \varepsilon_{0}$. Furthermore, it is shown in [10] that the Dirichlet-toNeumann operator $T$ depends analytically on variations of arbitrary smooth domains so that an alternative summation method such as Padé approximation can be effectively used to extend the convergence radius beyond $\varepsilon_{0}$.

\section{Spectral-Galerkin method}

We note that for each iteration $n$, Eq. (3.10) is simply the following Helmholtz equation in a separable elliptic domain:

$$
\begin{aligned}
& \partial_{\mu}^{2} U+\partial_{\theta}^{2} U+\frac{1}{2} k^{2} \cosh (2 \mu) U-\frac{1}{2} k^{2} \cos (2 \theta) U=F, \quad(\mu, \theta) \in \Omega_{a, b}, \\
& U(a, \theta)=\xi(\theta), \\
& \partial_{\mu} U(b, \theta)-T U(b, \theta)=\eta(\theta),
\end{aligned}
$$

with given $F(\mu, \theta), \xi(\theta)$ and $\eta(\theta)$. Hence, they can be efficiently solved by using a suitable spectral-Galerkin method which we describe below.

Thanks to the orthogonality of the angular Mathieu functions (2.13)-(2.14), we can expand $U(\mu, \theta), F(\mu, \theta), \xi(\theta)$ and $\eta(\theta)$ as

$$
\begin{aligned}
& (U(\mu, \theta), F(\mu, \theta))=\sum_{m=0}^{\infty}\left(\hat{u}_{1 m}(\mu), \hat{f}_{1 m}(\mu)\right) c e_{m}(\theta ; q)+\sum_{m=1}^{\infty}\left(\hat{u}_{2 m}(\mu), \hat{f}_{2 m}(\mu)\right) s e_{m}(\theta ; q), \\
& (\xi(\theta), \eta(\theta))=\sum_{m=0}^{\infty}\left(\hat{\xi}_{1 m}, \hat{\eta}_{1 m}\right) c e_{m}(\theta ; q)+\sum_{m=1}^{\infty}\left(\hat{\xi}_{2 m}, \hat{\eta}_{2 m}\right) s e_{m}(\theta ; q) .
\end{aligned}
$$

We recall that $c e_{m}$ and $s e_{m}$ satisfy the angular Mathieu equation (2.8):

$$
\begin{aligned}
& c e_{m}^{\prime \prime}+\left(\lambda_{c m}-2 q \cos (2 \theta)\right) c e_{m}=0, \\
& s e_{m}^{\prime \prime}+\left(\lambda_{s m}-2 q \cos (2 \theta)\right) s e_{m}=0 .
\end{aligned}
$$

Inserting the Mathieu expansions in (4.1a)-(4.1c) and using (2.16), we find that (4.1a)(4.1c) can be decomposed into the following sequence of one-dimensional problems ( $m=$ $0,1,2, \cdots)$ :

$$
\begin{aligned}
& \hat{u}_{1 m}^{\prime \prime}-\lambda_{c m} \hat{u}_{1 m}+\frac{1}{2} k^{2} \cosh (2 \mu) \hat{u}_{1 m}=\hat{f}_{1 m}, \quad \mu \in(a, b), \\
& \hat{u}_{1 m}(a)=\hat{\xi}_{1 m}, \quad \hat{u}_{1 m}^{\prime}(b)-\frac{M c_{m}^{\prime}(b ; q)}{M c_{m}(b ; q)} \hat{u}_{1 m}(b)=\hat{\eta}_{1 m},
\end{aligned}
$$

and

$$
\begin{aligned}
& \hat{u}_{2 m}^{\prime \prime}-\lambda_{s m} \hat{u}_{2 m}+\frac{1}{2} k^{2} \cosh (2 \mu) \hat{u}_{2 m}=\hat{f}_{2 m}, \quad \mu \in(a, b), \\
& \hat{u}_{2 m}(a)=\hat{\xi}_{2 m}, \quad \hat{u}_{2 m}^{\prime}(b)-\frac{M s_{m}^{\prime}(b ; q)}{M s_{m}(b ; q)} \hat{u}_{2 m}(b)=\hat{\eta}_{2 m},
\end{aligned}
$$


where $M c_{m}$ and $M s_{m}$ are the radial Mathieu-Hankel functions of the third kind.

We describe below a spectral-Galerkin method for (4.2) only, since (4.3) can be treated in exactly the same fashion. To this end, let us first make a change of variable $x=\frac{2(\mu-a)}{b-a}-1$ which maps $\mu \in(a, b)$ to $x \in I:=(-1,1)$. Denoting

$$
\begin{aligned}
& \tilde{u}_{1 m}(x)=\hat{u}_{1 m}(\mu), \quad \tilde{f}_{1 m}(x)=\hat{f}_{1 m}(\mu), \quad \tilde{\eta}_{1 m}=\hat{\eta}_{1 m}, \quad \tilde{\xi}_{1 m}=\hat{\xi}_{1 m}, \\
& t_{a}=\frac{2}{b-a}, \quad t_{b m}=-\frac{M c_{m}^{\prime}(b ; q)}{M c_{m}(b ; q)}, \quad \alpha=\frac{4}{(b-a)^{2}},
\end{aligned}
$$

Eq. (4.2) becomes

$$
\begin{aligned}
& \alpha \tilde{u}_{1 m}^{\prime \prime}-\lambda_{c m} \tilde{u}_{1 m}+q\left(e^{(b-a) x+(b+a)}+e^{-(b-a) x-(b+a)}\right) \tilde{u}_{1 m}=\tilde{f}_{1 m}, \quad x \in I, \\
& \tilde{u}_{1 m}(-1)=\tilde{\xi}_{1 m}, \quad t_{a} \tilde{u}_{1 m}^{\prime}(1)+t_{b m} \tilde{u}_{1 m}(1)=\tilde{\eta}_{1 m} .
\end{aligned}
$$

We first reformulate Eq. (4.4) into an equivalent problem with homogeneous boundary conditions. To this end, we set

$$
h_{1 m}(x)=\frac{\tilde{\eta}_{1 m}-t_{b m} \tilde{\xi}_{1 m}}{t_{a}+2 t_{b m}} x+\frac{\tilde{\eta}_{1 m}+t_{b m} \tilde{\xi}_{1 m}+t_{a} \tilde{\xi}_{1 m}}{t_{a}+2 t_{b m}}
$$

which satisfies the two boundary conditions in (4.4b). Hence, setting

$$
\begin{aligned}
& d=\frac{\tilde{\eta}_{1 m}-t_{b m} \hat{\xi}_{1 m}}{t_{a}+2 t_{b m}}, \quad f_{1 m}=\tilde{f}_{1 m}+\left[\lambda_{c m}-q\left(e^{(b-a) x+(b+a)}+e^{-(b-a) x-(b+a)}\right)\right] h_{1 m}, \\
& u_{1 m}(x)=\tilde{u}_{1 m}(x)+h_{1 m}(x)
\end{aligned}
$$

Eq. (4.4) becomes

$$
\begin{aligned}
& \alpha u_{1 m}^{\prime \prime}-\lambda_{c m} u_{1 m}+Q(x) u_{1 m}=f_{1 m}, \\
& u_{1 m}(-1)=0, \quad t_{a} u_{1 m}^{\prime}(1)+t_{b m} u_{1 m}(1)=0,
\end{aligned}
$$

where $Q(x)=q\left(e^{(b-a) x+(b+a)}+e^{-(b-a) x-(b+a)}\right)$.

Let $P_{N}$ be the space of complex-valued polynomials of degree less than or equal to $N$, and

$$
X_{N}^{(1, m)}:=\left\{u \in P_{N} \mid u(-1)=0, t_{a} u^{\prime}(1)+t_{b m} u(1)=0\right\} .
$$

The Spectral-Galerkin method for (4.5) is: find $u_{N}^{(1, m)} \in X_{N}^{(1, m)}$ such that

$$
\alpha \int_{I}\left(u_{N}^{(1, m)}\right)^{\prime \prime} \bar{v}_{N} d x+\int_{I}\left(Q-\lambda_{c m}\right) u_{N}^{(1, m)} \bar{v}_{N} d x=\int_{I} f_{1, m} \bar{v}_{N} d x, \quad \forall v_{N} \in X_{N}^{(1, m)},
$$

where $\bar{v}_{N}$ is the complex conjugate of $v_{N}$. We recall (cf. $[16,17]$ ) that if $t_{a}$ and $t_{b m}$ are real numbers and $P_{N}$ consists of real polynomials, then there exist unique real numbers $\left(\alpha_{k}^{(1, m)}, \beta_{k}^{(1, m)}\right)$ such that

$$
X_{N}^{(1, m)}=\operatorname{span}\left\{\gamma_{0}^{(1, m)}, \gamma_{1}^{(1, m)}, \cdots, \gamma_{N-2}^{(1, m)}\right\}
$$


where

$$
\gamma_{k}^{(1, m)}(x)=L_{k}(x)+\alpha_{k}^{(1, m)} L_{k+1}(x)+\beta_{k}^{(1, m)} L_{k+2}(x),
$$

and $L_{k}(x)$ is the $k$-th Legendre polynomial. It is easy to see that this is still true if we allow all of the $\left(\alpha_{k}^{(1, m)}, \beta_{k}^{(1, m)}\right), t_{a}, t_{b m}, P_{N}$, and $X_{N}^{(1, m)}$ to be complex valued. In fact, one easily verifies that

$$
\alpha_{k}^{(1, m)}=\frac{(2 k+3) t_{a}}{t_{a}(k+2)^{2}+2 t_{b m}}, \quad \beta_{k}^{(1, m)}=\alpha_{k}^{(1, m)}-1 .
$$

Now setting

$$
\begin{aligned}
& u_{N}^{(1, m)}(x):=\sum_{j=0}^{N-2} u_{j}^{(1, m)} \gamma_{j}(x), \quad \mathbf{u}:=\left(u_{0}^{(1, m)}, u_{1}^{(1, m)}, \cdots, u_{N-2}^{(1, m)}\right)^{T}, \\
& a_{j, n}^{(1, m)}:=\int_{I}\left(\gamma_{n}^{(1, m)}\right)^{\prime \prime} \bar{\gamma}_{j}^{(1, m)} d x, \quad A^{(1, m)}:=\left(a_{j, n}^{(1, m)}\right), \\
& b_{j, n}^{(1, m)}:=\int_{I} Q(x) \gamma_{n}^{(1, m)} \bar{\gamma}_{j}^{(1, m)} d x, \quad B^{(1, m)}:=\left(b_{j, n}^{(1, m)}\right), \\
& f_{j}^{(1, m)}=\int_{I} f_{1, m} \bar{\gamma}_{j}^{(1, m)} d x, \quad \mathbf{f}^{(1, m)}=\left(f_{0}^{(1, m)}, f_{1}^{(1, m)}, \cdots, f_{N-2}^{(1, m)}\right)^{T},
\end{aligned}
$$

the system (4.7) becomes the following complex-valued linear system:

$$
\left(\alpha A^{(1, m)}+B^{(1, m)}-\lambda_{c m} I\right) \mathbf{u}^{(1, m)}=\mathbf{f}^{(1, m)} .
$$

Due to the non-constant coefficients in $Q(x)$, the matrix $B^{(1, m)}$ is full. Since one has to solve the system (4.8) at each iteration $n$ of (3.10), it is more efficient to compute and store the $L U$ factorization of $\alpha A^{(1, m)}+B^{(1, m)}-\lambda_{c m} I$ and use it for each iteration.

Although the Mathieu functions have been frequently used by physicists and engineers, there were essentially no error estimates available in the context of spectral method for PDEs using Mathieu functions. Recently, Shen \& Wang [18] derived a first set of error estimates for the spectral-Galerkin method of the Helmholtz equation (with an approximate Dirichlet-to-Neumann operator, i.e., Eqs. (4.1a)-(4.1c) with $T$ being replaced by an approximated operator) using Mathieu functions. Let

$$
u_{N_{\mu}, N_{\theta}}(\mu, \theta)=\sum_{m=0}^{N_{\theta}}\left(\hat{u}_{N_{\mu}}^{1, m}(\mu) M c_{m}(\theta ; q)+\hat{u}_{N_{\mu}}^{2, m}(\mu) M s_{m}(\theta ; q)\right),
$$

where $u_{N_{\mu}}^{1, m}(\mu)$ and $u_{N_{\mu}}^{2, m}(\mu)$ are approximate solutions of (4.2) and (4.3) by the spectralGalerkin method described above. Then, based on the results in [18], it can be expected that the error $\left\|u-u_{N_{\mu}, N_{\theta}}\right\|_{L^{2}\left(\Omega_{a, b}\right)}$ will decay exponentially as $N_{\theta}, N_{\mu} \rightarrow \infty$. 


\section{Numerical results}

We now present some numerical results to demonstrate the robustness and effectiveness of the above scheme for the Helmholtz problem (2.17).

Before we present the numerical results, let us comment on the expected error estimates. Let $u$ be the exact solution of (3.8) and $u_{n}$ be the $n$-th term in its Taylor expansion (3.9). Given a triplet of discretization parameters $\left(M, N_{\mu}, N_{\theta}\right)$, let $u_{N_{\mu}, N_{\theta}}^{(n)}$ be the spectral approximation of $u_{n}$ defined in (4.9). Then, the final approximation of $u$ is

$$
u_{N_{\mu}, N_{\theta}}^{M}(\mu, \theta)=\sum_{n=0}^{M} u_{N_{\mu}, N_{\theta}}^{(n)}(\mu, \theta) \varepsilon^{n} .
$$

Writing

$$
u-u_{N_{\mu}, N_{\theta}}^{M}=\left(u-\sum_{n=0}^{M} u_{n} \varepsilon^{n}\right)+\sum_{n=0}^{M}\left(u_{n}-u_{N_{\mu}, N_{\theta}}^{(n)}\right) \varepsilon^{n},
$$

and using the triangular inequality, we can expect, for smooth perturbation $f(\theta)$, an error estimate of the form

$$
\left\|u-u_{N_{\theta}, N_{\mu}}^{M}\right\|_{L^{2}\left(\Omega_{a, b}\right)} \lesssim \varepsilon^{M}+\exp \left(-\alpha N_{\mu}\right)+\exp \left(-\beta N_{\theta}\right),
$$

where $\alpha, \beta$ are some positive numbers. A complete proof of this result is highly nontrivial due to the complexity of the algorithm so we shall only provide some numerical verifications. In the following, we shall present numerical results which are consistent with the above expected error estimate.

\subsection{Test with exact solutions}

It is well known that the Helmholtz equation (2.17a) admits a family of exact solutions:

$$
v(\mu, \theta)=M c_{m}(\mu ; q) c e_{m}(\theta ; q) \quad \text { or } \quad M s_{m}(\mu ; q) s e_{m}(\theta ; q), \quad m=0,1,2, \cdots,
$$

where, we recall, $q=k^{2} / 4$ with $k$ being the wave number in (3.10).

To validate the spectral-Galerkin method in Section 4, we first preform a set of tests on a regular elliptical obstacle, i.e., $g(\theta) \equiv 0$. Thanks to the orthogonality of the angular Mathieu functions, it is clear that $M c_{m}$ and $M s_{m}$ are respectively solutions of (4.2) and (4.3) with $f_{1 m}=f_{2 m}=0$ and $\hat{\eta}_{1 m}=\hat{\eta}_{2 m}=0$. So we just need to test the one dimensional solver for (4.2) and (4.3). We take $a=1, b=2$ and the exact solution of (4.2) to be $M c_{2}(\mu ; q)$. In Fig. 1 , we plot the $L^{2}$-error vs. wave numbers with different number of modes $N_{\mu}$. We observe that for fixed wave number $k$, the error converges exponentially as soon as enough modes are used.

In the next set of tests, we show that the transformed field expansion algorithm described in Section 3 is robust and efficient. We consider two elongated obstacles (cf. Fig. 2) described by

$$
f(\theta)=\sin (\theta) \text { and } f(\theta)=\frac{3}{2} \cos ^{2}(\theta)-\frac{1}{2}
$$




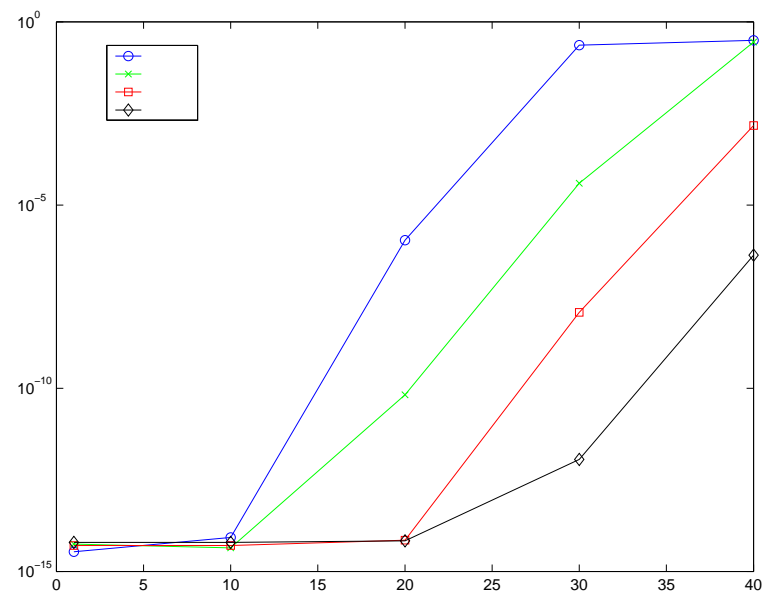

Figure 1: $L^{2}$-error vs. the number of modes $N_{\mu}$ for different wave numbers.
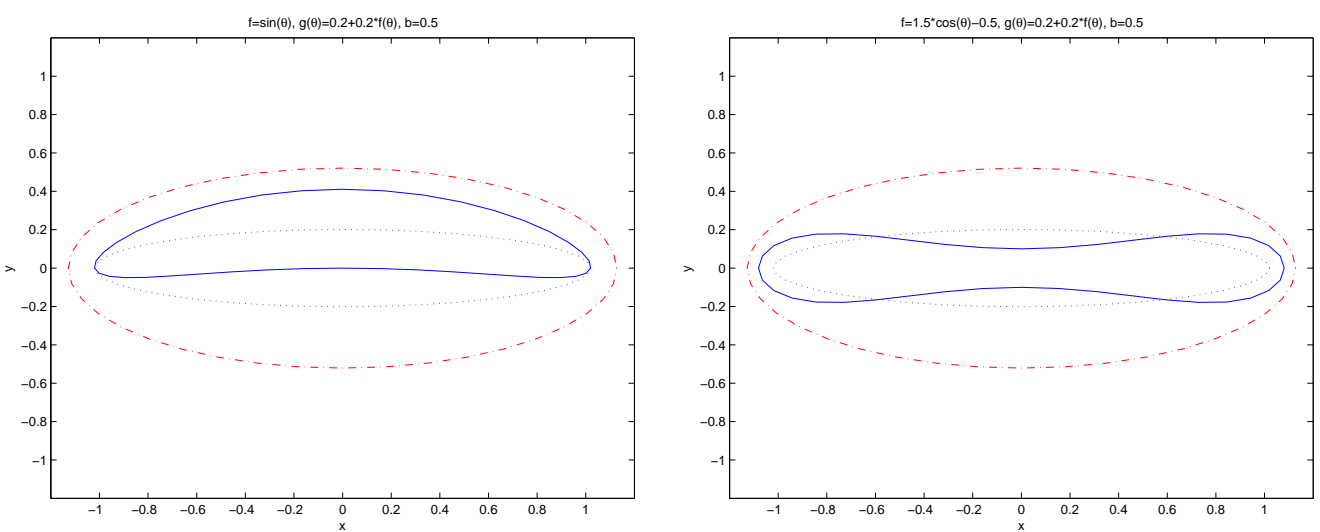

Figure 2: Geometries of scattering configurations: the solid lines describe the obstacles as perturbations of profiles given by the dotted lines; the dashed lines represent the artificial boundaries.
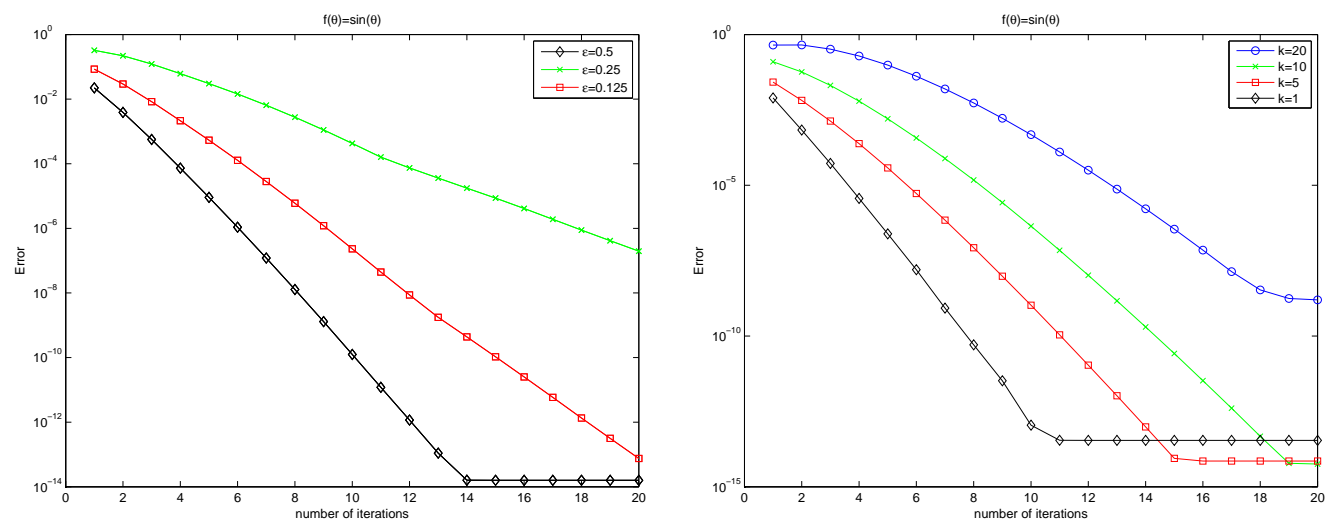

Figure 3: Convergence history of the TFE algorithm with $f(\theta)=\sin (\theta), a=1, b=2$ and a fixed resolution $N_{\mu}=40, N_{\theta}=50$. 
Table 1: Comparison of errors for fixed $a=1$ and different $b$.

\begin{tabular}{|c|c|c|c|}
\hline & Case I. & $a=1.0, b=2.0$ & \\
\hline$N_{\mu}$ & $k=10$ & $k=20$ & $k=30$ \\
\hline 20 & $3.19 \mathrm{E}-004$ & N/A & $\begin{array}{l}\mathrm{N} / \mathrm{A} \\
\end{array}$ \\
\hline 30 & 8.78E-009 & $2.66 \mathrm{E}-002$ & $\mathrm{~N} / \mathrm{A}$ \\
\hline 40 & $3.49 \mathrm{E}-014$ & 4.08E-006 & $\mathrm{N} / \mathrm{A}$ \\
\hline 50 & 7.44E-015 & $3.86 \mathrm{E}-010$ & $1.08 \mathrm{E}-004$ \\
\hline \multirow[t]{2}{*}{60} & 7.45E-015 & 9.45E-015 & 7.59E-008 \\
\hline & Case II. & $a=1.0, b=1.7$ & \\
\hline$N_{\mu}$ & $k=10$ & $k=20$ & $k=30$ \\
\hline 10 & $1.75 \mathrm{E}-002$ & N/A & N/A \\
\hline 16 & $1.24 \mathrm{E}-005$ & 0.118 & N/A \\
\hline 24 & $1.79 \mathrm{E}-010$ & 5.44E-005 & 0.126 \\
\hline 32 & 8.12E-015 & $9.11 \mathrm{E}-009$ & $1.15 \mathrm{E}-004$ \\
\hline \multirow[t]{2}{*}{40} & 8.17E-015 & $3.37 \mathrm{E}-013$ & $6.36 \mathrm{E}-008$ \\
\hline & Case III. & $a=1.0, b=1.4$ & \\
\hline$N_{\mu}$ & $k=10$ & $k=20$ & $k=30$ \\
\hline 10 & $1.98 \mathrm{E}-005$ & $1.96 \mathrm{E}-002$ & $\mathrm{~N} / \mathrm{A}$ \\
\hline 16 & $4.12 \mathrm{E}-010$ & 7.97E-006 & $2.17 \mathrm{E}-003$ \\
\hline 24 & 7.90E-015 & $3.69 \mathrm{E}-011$ & $1.61 \mathrm{E}-007$ \\
\hline \multirow[t]{2}{*}{32} & 8.01E-015 & 5.72E-015 & $1.59 \mathrm{E}-012$ \\
\hline & Case IV. & $a=1.0, b=1.11$ & \\
\hline$N_{\mu}$ & $k=10$ & $k=20$ & $k=30$ \\
\hline 10 & $6.36 \mathrm{E}-011$ & $7.24 \mathrm{E}-008$ & $5.56 \mathrm{E}-006$ \\
\hline 16 & $5.48 \mathrm{E}-015$ & $3.65 \mathrm{E}-014$ & 2.63E-011 \\
\hline 24 & $5.49 \mathrm{E}-015$ & $1.38 \mathrm{E}-014$ & $1.39 \mathrm{E}-013$ \\
\hline 32 & $5.56 \mathrm{E}-015$ & $9.25 \mathrm{E}-015$ & $1.39 \mathrm{E}-013$ \\
\hline
\end{tabular}

Two sets of numerical tests are conducted:

(i) Fix $k=1$ and vary $\varepsilon$ through the values $\varepsilon=0.125,0.25,0.5$.

(ii) Fix $\varepsilon=0.1$ and vary the wave number $k$ among $k=1,5,10,20$.

In Fig. 3, we present the results for $f(\theta)=\sin (\theta)$ with $a=1, b=2$ and a fixed resolution $N_{\mu}=40, N_{\theta}=50$. We observe that, for both fixed wave number $k=1$ and fixed perturbation parameter $\varepsilon=0.1$, the TFE algorithm converges monotonically for all cases, and it achieves the accuracy limited by discretization parameters $N_{\mu}$ and $M$. Similar convergence behavior is observed for the obstacle described by $f(\theta)=\frac{3}{2} \cos ^{2}(\theta)-\frac{1}{2}$.

A distinct advantage of the TFE algorithm is that we can choose the artificial boundary as close to the obstacle as possible, since we use a spectral approximation of the exact Dirichlet-to-Neumann operator as boundary conditions on the artificial boundary. Thus, the effective wave number in the radial direction is reduced from $(a+\max |g|) k$ to essentially $(\max |g|) k$, and consequently, the number of modes needed in the radial direction can be reduced significantly. To illustrate this property, we fix $\varepsilon=0.1, a=1$ and take $b=$ $2.0,1.7,1.4,1.11$. The results are reported in Table 1 . We observe, for example, that for $b=2.0$ and $k=10$, we need $N_{\mu}=50$ to achieve the near machine accuracy of $10^{-15}$, 

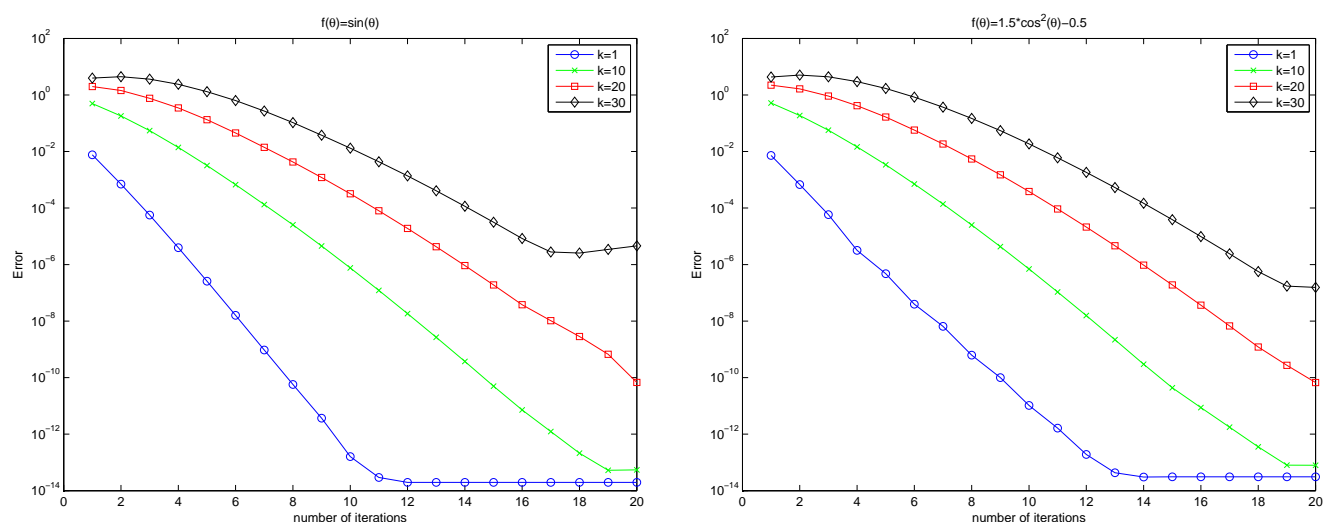

Figure 4: Convergence history of the TFE algorithm for plane wave scattering: $\varepsilon=0.1, a=1, b=1.2$ with $N_{\mu}=50$ and $N_{\theta}=70$. Left, $f(\theta)=\sin (\theta)$; Right, $f(\theta)=\frac{3}{2} \cos ^{2}(\theta)-\frac{1}{2}$.

while only $N_{\mu}=32, N_{\mu}=24, N_{\mu}=16$ are needed for $b=1.7, b=1.4, b=1.11$, respectively. Thus, choosing $b$ close to $a+\max |g|$ allows us to use much less points in the radial direction.

\subsection{Plane-wave scattering}

Here we are interested in computed the scattered field from a plane-wave incident upon the obstacle depicted in Fig. 2. Since we no longer have an exact solution, for comparison purposes, we use a high resolution approximation $\left(N_{\theta}=200, N_{r}=80\right)$ as the reference solution.

In Fig. 4, we present the convergence history of the TFE algorithm for the plane wave scattering upon the two obstacles described by (5.3) and $\varepsilon=0.1, a=1, b=1.2$ with $N_{\mu}=50$ and $N_{\theta}=70$. We observe that the convergence behavior for the plane wave scattering is essentially the same as for the case with an exact solution. Namely, it converges monotonically with respect to the iteration number and the achievable accuracy is only limited by the discretization parameters $N_{\mu}$ and $N_{\theta}$.

\section{Concluding remarks}

We developed in this paper an efficient and accurate method for solving the two dimensional Helmholtz equation in domains exterior to elongated obstacles. The method is based on the transformed field expansion (TFE) which has been successfully used in $[5,9]$ previously for solving the acoustic scattering problems in both two-dimensional circular and three-dimensional spherical domains. However, these algorithms may become inefficient for elongated obstacles. Therefore, we considered a spectral approximation based the Mathieu functions which arise naturally in separation of variables for Helmholtz equations in 2-D elliptical domains. 
It turned out that the use of elliptic transform and Mathieu functions introduces significant complications in the algorithm development and implementation of the TFE method. With delicate analytical derivations and careful manipulations of the Mathieu functions in programming, we have successful derived the TFE algorithm under elliptical coordinates and developed a stable implementation of the spectral-Galerkin method using Mathieu functions.

The illustrative numerical results presented in this paper indicate that the TFE algorithm with a spectral-Galerkin solver using Mathieu functions is efficient and accurate for acoustic scattering from elongated obstacles.

Acknowledgments The work of Q. Fang and J. Shen is supported in part by NSF grant DMS-0610646, while the work of L. Wang is partially supported by AcRF Tier 1 Grant RG58/08, Singapore MOE Grant T207B2202, and Singapore NRF2007IDM-IDM002-010.

\section{References}

[1] F. A. Alhargan. Algorithm for the computation of all Mathieu functions of integer orders. ACM Trans. Math. Softw., 26:390-407, 2001.

[2] Oscar P. Bruno and Fernando Reitich. Solution of a boundary value problem for the Helmholtz equation via variation of the boundary into the complex domain. Proc. Roy. Soc. Edinburgh Sect. A, 122(3-4):317-340, 1992.

[3] Oscar P. Bruno and Fernando Reitich. Numerical solution of diffraction problems: A method of variation of boundaries. J. Opt. Soc. Am. A, 10(6):1168-1175, 1993.

[4] Oscar P. Bruno and Fernando Reitich. Numerical solution of diffraction problems: A method of variation of boundaries. II. Finitely conducting gratings, Padé approximants, and singularities. J. Opt. Soc. Am. A, 10(11):2307-2316, 1993.

[5] Q. Fang, D. Nicholls, and Jie Shen. A stable, high-order method for two-dimensional bounded-obstacle scattering. J. Comput. Phys., 224:1145-1169, 2007.

[6] Marcus J. Grote and Joseph B. Keller. On nonreflecting boundary conditions. J. Comput. Phys., 122(2):231-243, 1995.

[7] A. Linder and H. Freese. A new method to compute Mathieu functions. J. Phys. A, 27:5565$5571,1994$.

[8] N. W. McLachlan. Theory and applications of Mathieu functions. Oxford Press, London, 1951.

[9] D. Nicholls and Jie Shen. A stable, high-order method for two-dimensional boundedobstacle scattering. SIAM J. Sci. Comput., 28:1398-1419, 2006.

[10] David P. Nicholls and Fernando Reitich. Analytic continuation of Dirichlet-Neumann operators. Numer. Math., 94(1):107-146, 2003.

[11] David P. Nicholls and Fernando Reitich. Shape deformations in rough surface scattering: Cancellations, conditioning, and convergence. J. Opt. Soc. Am. A, 21(4):590-605, 2004.

[12] David P. Nicholls and Fernando Reitich. Shape deformations in rough surface scattering: Improved algorithms. J. Opt. Soc. Am. A, 21(4):606-621, 2004.

[13] Lord Rayleigh. On the dynamical theory of gratings. Proc. Roy. Soc. London, A79:399-416, 1907.

[14] F. Reitich and K. Tamma. State-of-the-art, trends, and directions in computational electromagnetics. CMES Comput. Model. Eng. Sci., 5(4), 2004.

[15] S. O. Rice. Reflection of electromagnetic waves from slightly rough surfaces. Comm. Pure Appl. Math., 4:351-378, 1951. 
[16] Jie Shen. Efficient spectral-Galerkin method I. direct solvers for second- and fourth-order equations by using Legendre polynomials. SIAM J. Sci. Comput., 15:1489-1505, 1994.

[17] Jie Shen. Efficient Chebyshev-Legendre Galerkin methods for elliptic problems. In A. V. Ilin and R. Scott, editors, Proceedings of ICOSAHOM'95, pages 233-240. Houston J. Math., 1996.

[18] Jie Shen and Li-Lian Wang. On spectral approximations in elliptical geometries using Mathieu functions. To appear in Math. Comp., 2008.

[19] Karl F. Warnick and Weng Cho Chew. Numerical simulation methods for rough surface scattering. Waves Random Media, 11(1):R1-R30, 2001. 